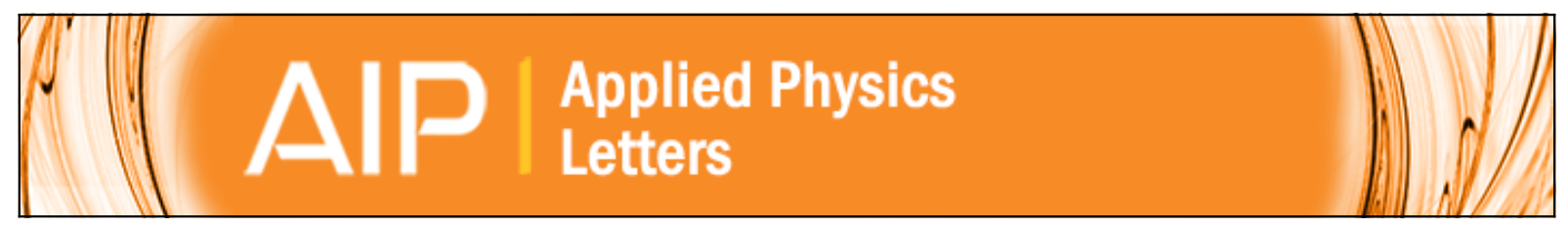

\title{
Current-induced cleaning of graphene
}

J. Moser, A. Barreiro, and A. Bachtold

Citation: Applied Physics Letters 91, 163513 (2007); doi: 10.1063/1.2789673

View online: http://dx.doi.org/10.1063/1.2789673

View Table of Contents: http://scitation.aip.org/content/aip/journal/apl/91/16?ver=pdfcov

Published by the AIP Publishing

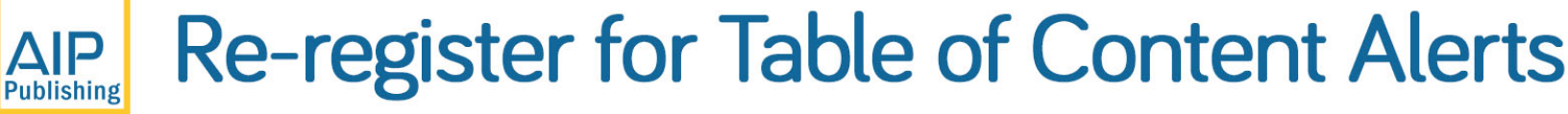

\section{Create a profile. \\ Sign up today!}




\title{
Current-induced cleaning of graphene
}

\author{
J. Moser, ${ }^{\text {a) }}$ A. Barreiro, and A. Bachtold ${ }^{\text {b) }}$ \\ CIN2, Universitat Autonoma de Barcelona, E-08193 Bellaterra, Spain \\ and CNM-CSIC, Universitat Autonoma de Barcelona, E-08193 Bellaterra, Spain
}

(Received 2 August 2007; accepted 4 September 2007; published online 19 October 2007)

\begin{abstract}
A simple yet highly reproducible method to suppress contamination of graphene at low temperature inside the cryostat is presented. The method consists of applying a current of several milliamperes through the graphene device, which is here typically a few microns wide. This ultrahigh current density is shown to remove contamination adsorbed on the surface. This method is well suited for quantum electron transport studies of undoped graphene devices, and its utility is demonstrated here by measuring the anomalous quantum Hall effect. (C) 2007 American Institute of Physics.
\end{abstract}

[DOI: $10.1063 / 1.2789673$ ]

The discovery of graphene, a one-atom thick layer of graphite, has generated considerable interest by opening avenues in condensed matter physics. ${ }^{1}$ Graphene is an appealing system for basic research due to its unusual electronic properties; namely, charge carriers behave like chiral massless Dirac particles. ${ }^{2-5}$ The material also holds promise for technological applications, such as in nanoelectronics; ${ }^{6}$ in addition, graphene has been shown to be an outstanding gas sensor able to detect minute amounts of molecular dopants. ${ }^{7,8}$ Yet, what makes graphene an enticing material is also the source of great technological difficulties: being in essence a surface, graphene proves to be extremely sensitive to contamination.

Recently, different approaches have been put forward to address this problem. Annealing at several hundreds of Celsius in ultrahigh vacuum or in $\mathrm{Ar} / \mathrm{H}_{2}$ environment has been shown to remove contamination. ${ }^{9,10}$ Unfortunately, however, such annealing processes are difficult to implement in a cryostat for low-temperature measurements, and transferring samples from the annealing chamber to the cryostat is not convenient, since exposure to air reintroduces contamination.

In this letter, a simple yet highly reproducible method to suppress contamination at low temperature inside the cryostat is presented. The method is based on the application of a large current through the graphene device generating several tens of milliwatts dissipation over a few $\mu \mathrm{m}^{2}$ large surface. Remarkably, graphene can sustain such extreme conditions while the adsorbed contamination gets removed. We employ atomic force microscopy (AFM) to show how adsorbates, as well as intentionally deposited CdSe particles, melt away. These cleaned graphene devices allow us to measure the anomalous quantum Hall effect.

We start by briefly describing our fabrication technique. Graphene flakes are deposited by mechanical exfoliation of kish graphite (Toshiba Ceramics) on degenerately doped silicon substrates coated with $270 \mathrm{~nm}$ of thermal silicon oxide. We use standard wafer protection tape as it leaves little adhesive residue on substrates. $\mathrm{Cr} / \mathrm{Au}$ electrodes are fabricated on top of the samples by electron beam lithography followed by lift-off in acetone and dicholoroethane. Two-point dc transport measurements are conducted both at room temperature in air for AFM imaging and in a $\mathrm{He}^{4}$ cryostat.

\footnotetext{
${ }^{a)}$ Electronic mail: moser.joel@gmail.com

b) Electronic mail: adrian.bachtold@cnm.es
}

Graphene is able to carry very high electrical currents without sustaining damage. Applying a source-drain bias $V$ of a few volts across the sample induces a large current flow $I$ of a few milliamperes, as shown in Fig. 1. Taking the sample width of $4 \mu \mathrm{m}$ and a thickness of $0.35 \mathrm{~nm}$, this translates into an extremely large current density $J$ of a few $10^{8} \mathrm{~A} / \mathrm{cm}^{2}$. For comparison, $J$ is only a few times larger in carbon nanotubes, ${ }^{11,12}$ and for both materials, $J$ is several orders of magnitude larger than in present-day interconnects.

The effects of a large electrical current passing through a mesoscopic, conducting device include electromigration ${ }^{13,14}$ and Joule heating. We expect that the large power $P$ $\simeq 20 \mathrm{~mW}$ dissipated over a small area of a few $\mu \mathrm{m}^{2}$ significantly increases the sample temperature $T$. In order to show this, we monitor the four-point resistance $R$ of a gold strip $(R \simeq 60 \Omega$ at room $T)$ fabricated on the substrate $30 \mu \mathrm{m}$ away from the graphene sample. Having initially calibrated $R$ against a commercial thermometer in thermal equilibrium, we estimate that the temperature rises by $\sim 25 \mathrm{~K}$ at the gold strip when a power of $34 \mathrm{~mW}$ is dissipated in the graphene sample at $4.2 \mathrm{~K}$. However, an estimate of the graphene temperature is difficult to obtain, since most of the heat is evacuated through the $\mathrm{Au}$ electrodes that are thermally anchored to

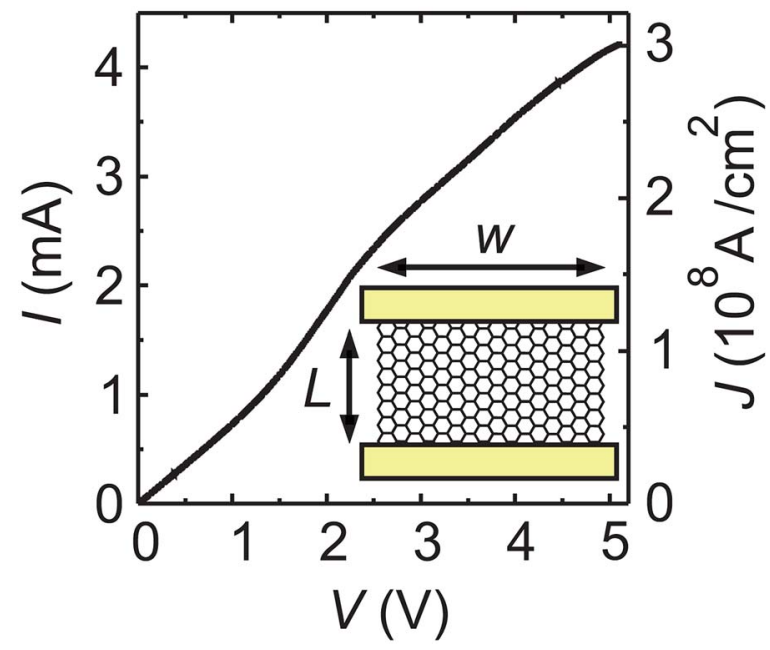

FIG. 1. (Color online) current $I$ (left axis) and current density $J$ (right axis) as a function of source-drain bias $V$ applied across the graphene sample in helium atmosphere at $T=76 \mathrm{~K}$. Graphene width $w=4 \mu \mathrm{m}$ and interelectrode distance $L=1 \mu \mathrm{m}$. Inset: schematic of the device. 

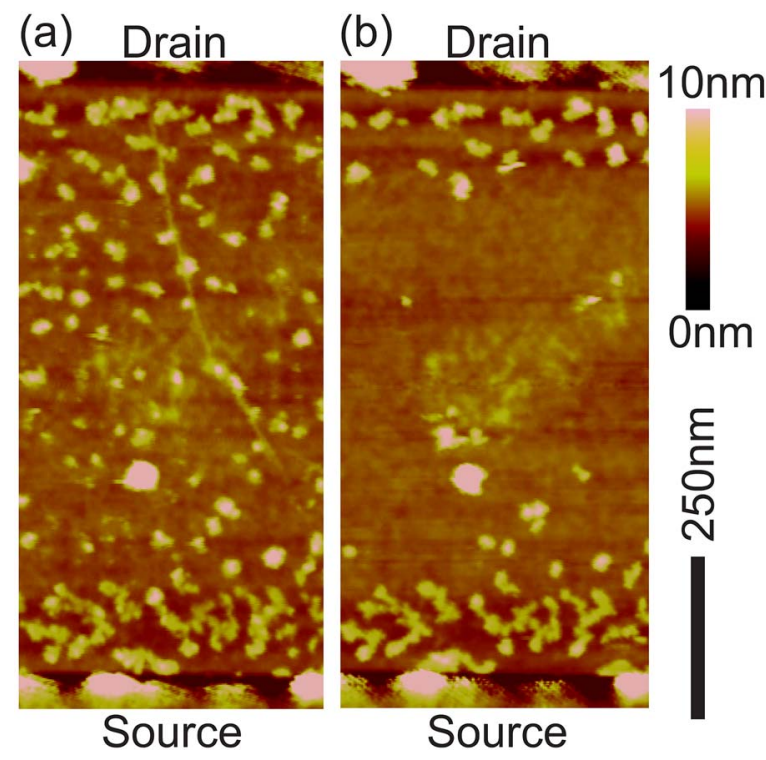

FIG. 2. (Color online) CdSe nanoparticles are deposited on a graphene sample, and imaged by AFM before [Fig. 2(a)] and after [Fig. 2(b)] applying a large electrical current between source and drain. Both figures are topographic scans. Figure 2(b) indicates that most nanoparticles have vanished from the area located halfway between the source and the drain. We imaged the entire sample and verified that particles did not form clusters and did not migrate to the edges.

the liquid helium bath (see below). Further insight is gained by depositing $\mathrm{CdSe}$ nanoparticles with a mean diameter of $5 \mathrm{~nm}$ on a graphene sample and applying a large current. Figure 2 presents topographic AFM scans of the sample before [Fig. 2(a)] and after [Fig. 2(b)] the current flow. Figure 2(b) shows that most nanoparticles have vanished. Owing to high temperatures generated by the large Joule heating, nanoparticles might have undergone various phase transitions: they might have melted and formed a thin film over the sample, or they might also have evaporated or sublimated. In addition, each of these mechanisms may be assisted by electromigration. ${ }^{15} \mathrm{~A}$ rough estimate for the temperature reached is given by the melting temperature of $5 \mathrm{~nm}$ diameter CdSe particles, which is expected to be $\sim 600{ }^{\circ} \mathrm{C} .{ }^{16} \mathrm{Fu}-$ ture work should help identify the mechanism by which nanoparticles disappear and allow for a better estimate of the graphene temperature. Eventually, note that the presence of the remaining particles close to the electrodes suggests that these act as heat sinks [Fig. 2(b)].

In the following, we demonstrate how this effect can be harnessed to remove contaminants from graphene samples. AFM imaging reveals the influence of the large electrical current on the morphology of the sample surface. Figures 3(a)-3(c) display AFM scans for the signal amplitude at various stages of the cleaning process. Typically, freshly deposited graphene sheets have an average roughness $R_{a}$ $\simeq 0.1 \mathrm{~nm}$. Right after fabrication [Fig. 3(a)], $R_{a} \simeq 0.4 \mathrm{~nm}$ is measured, suggesting the presence of contamination on top of the graphene. Figure 3(b) captures an intermediate state obtained by ramping $V$ to $3 \mathrm{~V}$. Contaminants migrate and cluster up. As $V$ is further increased, the sample area halfway between source and drain becomes almost as smooth as the substrate [Fig. 3(c), for $V=4.6 \mathrm{~V}$ ] and the roughness is down to $R_{a} \simeq 0.1 \mathrm{~nm}$, as before fabrication. Figure 3(c) also shows that some contamination has accumulated at the corner of the
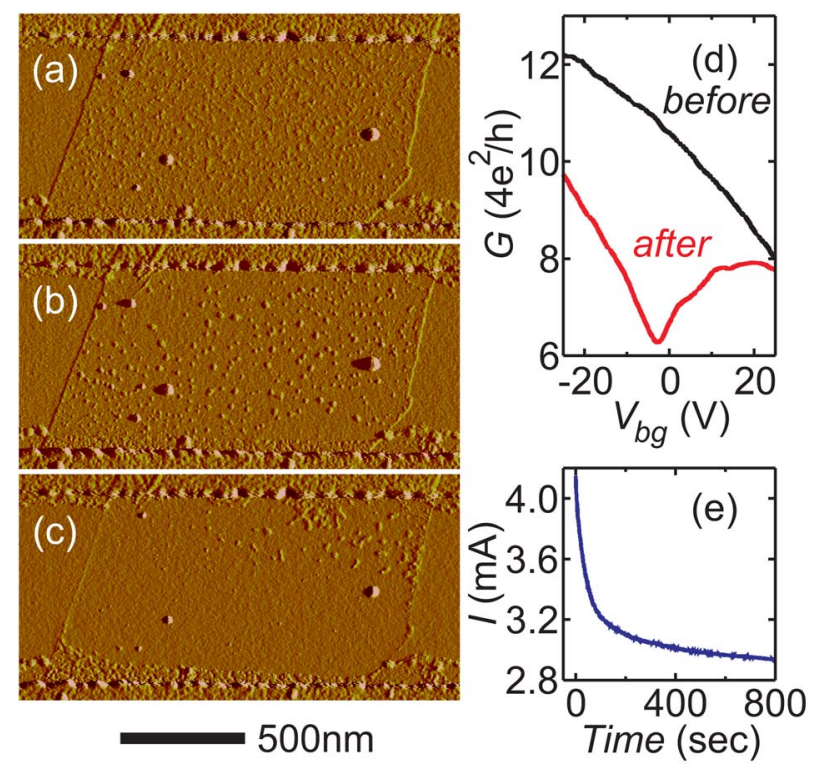

FIG. 3. (Color online) [(a)-(c)] AFM signal amplitude scans captured at various stages of the current-induced cleaning process in air. (d) Two-point conductance $G$ as a function of backgate bias $V_{\text {bg }}$ before and after the application of a large current in helium atmosphere and at $T=76 \mathrm{~K}$. (e) Time dependence of current $I$ at fixed bias $V=5 \mathrm{~V}$.

device (such a migration is not observed with CdSe particles).

This contamination removal has drastic effects on the sample doping. Electric field effect can be used to dope graphene either with electrons or holes, resulting in a minimum conductivity at the charge neutrality point. The black curve in Fig. 3(d) represents the low-bias conductance $G$ as a function of a backgate bias $V_{\mathrm{bg}}$ applied to the silicon substrate, for the same sample as in Fig. 1 but prior to any large current flow. Measurements are performed under helium atmosphere at $T=76 \mathrm{~K}$ in a cryostat. $G$ decreases continuously as a function of $V_{\mathrm{bg}}$, which indicates that the conductance minimum is beyond the voltage range and that doping is important. After high-bias treatment we observe a welldefined conductance minimum $G_{\min }$ close to zero $V_{\mathrm{bg}}$ that denotes the reduction of doping.

The strong shift of $G_{\min }$ to zero $V_{\mathrm{bg}}$, along with the recovered smoothness of the sample, indicates that unavoidable fabrication residues act as dopants that are removed upon passing a large electrical current through the sample. These residues may consist in part of nondissolved polymethyl methacrylate (PMMA). ${ }^{9}$ Note that in air, this cleaning process tends to be counterbalanced by the doping effect of other molecular adsorbates (e.g., oxygen), as a result of which $G_{\min }$ is found to slowly drift in time back to large, positive $V_{\text {bg }}$ values.

Special care has to be taken to preserve the integrity of the device upon applying large source-drain biases; we proceed as follows. Setting $V_{\mathrm{bg}}=0$, we slowly raise $V$. We regularly hold $V$ constant to monitor the time dependence of the current $I$. If $I$ stays constant, we keep on increasing $V$. This is repeated until $I$ continuously decreases as a function of time and saturates [see Fig. 3(e) for a fixed $V=5 \mathrm{~V}$, sample of Figs. 1 and 3(d)]. The continuous decay of $I$ in time at fixed $V$ and $V_{\mathrm{bg}}=0$ signals a smooth shift of $G_{\min }$ to zero $V_{\mathrm{bg}}$. Note that an acceleration of the current decay usually precedes the rupture of graphene. 


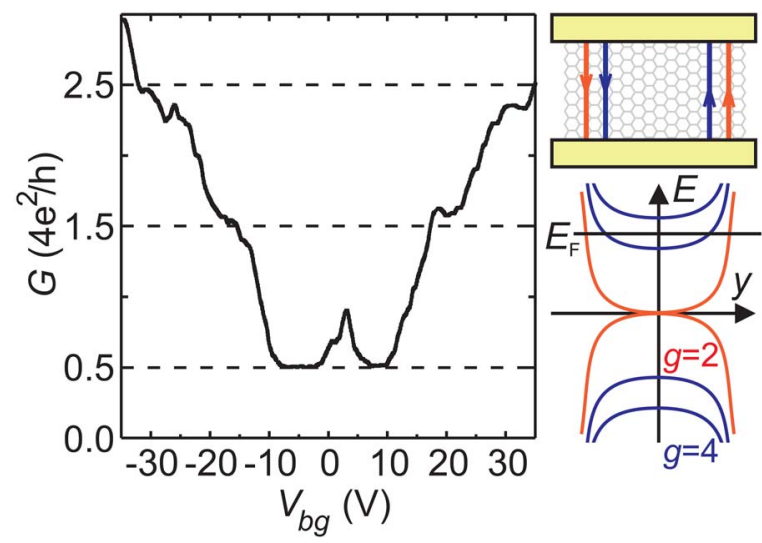

FIG. 4. (Color online) (Left) Two-point conductance $G\left(V_{\mathrm{bg}}\right)$ at $B=9 \mathrm{~T}$ and $T=4.2 \mathrm{~K}$ revealing the anomalous quantum Hall effect. (Right) Edge channels in ideally clean, single layer graphene. Electron and holelike channels coexist close to the neutrality point, yielding a channel degeneracy $g=2$. The $y$ axis is along the sample width. Levels are bent at the edges due to hardwall confinement.

Remarkably, this simple cleaning technique allows us to observe the anomalous, half-integer quantum Hall effect ${ }^{2,3}$ that is the signature of a clean, single graphene layer. In graphene, the $n$th Landau level (LL) energy reads $E_{n}$ $=\operatorname{sgn}(n) v_{F} \sqrt{2 e \hbar|n| B}$, where $n$ is the electron or hole LL index and $v_{F} \simeq 10^{6} \mathrm{~m} \mathrm{~s}^{-1}$ is the Fermi velocity. Edge channels have a degeneracy $g=4$ that accounts for both spin and sublattice degeneracies. The presence of electron and hole edge channels at the neutrality point (LL index $n=0$ ) reduces the number of current-carrying modes by half, yielding $g=2$ (see schematic of Fig. 4.) The two-point Landauer conductance reads $G=\Sigma g e^{2} / h$, where the sum is carried over all the levels, and is therefore quantized in $4 e^{2} / h(n+1 / 2)$ as $V_{\mathrm{bg}}$ is swept. ${ }^{17}$ Figure 4 shows a clear quantization at $1 / 2 \times 4 e^{2} / \mathrm{h}$, whereas the other plateaus are not as well defined (the same is observed for five other samples). The robustness of the first plateau is explained by the large energy gaps $\left|E_{ \pm 1}-E_{0}\right|$ $\simeq 1200 \mathrm{~K}$ at $B=9 \mathrm{~T}$. Importantly, the plateau is visible only after the current-induced cleaning process. This indicates that our process enhances the mobility of the sample and/or improves the homogeneity of the doping over the device area.

To conclude, we have demonstrated a simple, yet highly reproducible technique to obtain clean graphene devices out of initially highly contaminated samples. Our method takes advantage of the large electrical current density that graphene can sustain to remove contaminants from the sample surface. Compared to other methods, the reported technique has the advantage to be operative at low $T$ inside a cryostat, without having to cycle the cryostat to room $T$. In addition, we avoid contamination in air that would naturally occur upon transferring the device from the annealing chamber to the cryostat.

We thank P. Jarillo-Herrero for showing us the mechancal exfoliation technique. This work was supported by an EURYI grant and FP6-IST-021285-2.

${ }^{1}$ For a review, see: A. Castro Neto, F. Guinea, and N. M. Peres, "Drawing conclusions from graphene," Phys. World, November (2006). A. K. Geim and K. S. Novoselov, Nat. Mater. 6, 183 (2007); M. I. Katsnelson, Mater. Today 10, 20 (2007).

${ }^{2}$ K. S. Novoselov, A. K. Geim, S. V. Morozov, D. Jiang, M. I. Katsnelson, I. V. Grigorieva, S. V. Dubonos, and A. A. Firsov, Nature (London) 438, 197 (2005).

${ }^{3}$ Y. Zhang, Y.-W. Tan, H. L. Stormer, and P. Kim, Nature (London) 438, 201 (2005).

${ }^{4}$ V. P. Gusynin and S. G. Sharapov, Phys. Rev. Lett. 95, 146801 (2005).

${ }^{5}$ N. M. R. Peres, F. Guinea, and A. H. Castro Neto, Phys. Rev. B 73, 125411 (2006).

${ }^{6}$ C. Berger, Z. Song, X. Li, X. Wu, N. Brown, C. Naud, D. Mayou, T. Li, J. Hass, A. N. Marchenkov, E. H. Conrad, P. N. First, and W. A. de Heer, Science 312, 1191 (2006).

${ }^{7}$ F. Schedin, A. K. Geim, S. V. Morozov, E. H. Hill, P. Blake, M. I. Katsnelson, and K. S. Novoselov, Nat. Mater. 6, 652 (2007).

${ }^{8}$ E. H. Hwang, S. Adam, S. Das Sarma, and A. K. Geim, e-print arXiv:cond-mat/0610834v1.

${ }^{9}$ M. Ishigami, J. H. Chen, W. G. Cullen, M. S. Fuhrer, and E. D. Williams, Nano Lett. 7, 1643 (2007).

${ }^{10}$ E. Stolyarova, K. T. Rim, S. Ryu, J. Maultzsch, P. Kim, L. E. Brus, T. F. Heinz, M. S. Hybertsen, and G. W. Flynn, Proc. Natl. Acad. Sci. U.S.A. 104, 9209 (2007).

${ }^{11}$ Z. Yao, C. L. Kane, and C. Dekker, Phys. Rev. Lett. 84, 2941 (2000).

${ }^{12}$ B. Bourlon, D. C. Glattli, B. Plaçais, J. M. Berroir, C. Miko, L. Forró, and A. Bachtold, Phys. Rev. Lett. 92, 026804 (2004).

${ }^{13}$ R. E. Hummel, Int. Mater. Rev. 39, 97 (1994).

${ }^{14}$ H. Park, A. K. L. Lim, J. Park, A. P. Alivisatos, and P. L. McEuen, Appl. Phys. Lett. 75, 301 (1999).

${ }^{15}$ B. C. Regan, S. Aloni, R. O. Ritchie, U. Dahmen, and A. Zettl, Nature (London) 428, 924 (2004).

${ }^{16}$ The melting temperature $T_{m}$ of $4 \mathrm{~nm}$ diameter CdS particles has been measured to be $1180 \mathrm{~K}$ [A. N. Goldstein, C. M. Echer, and A. P. Alivisatos, Science 256, 1425 (1992)]. $T_{m}$ of CdSe particles can be roughly estimated using $T_{m} / T_{m}^{\text {bulk }}=1-C / d$, where $d$ is the particle diameter and $T_{m}^{\text {bulk }}$ is the bulk material melting temperature $(1510 \mathrm{~K}$ for CdSe and $2020 \mathrm{~K}$ for CdS) [Z. Zhang, M. Zhao, and Q. Jiang, Semicond. Sci. Technol. 16, L33 (2001)]. $C$ is material dependent; yet, here, we take the same value for CdSe and CdS.

${ }^{17}$ H. B. Heersche, P. Jarillo-Herrero, J. B. Oostinga, L. M. K. Vandersypen, and A. F. Morpurgo, Nature (London) 446, 56 (2007). 